\title{
Enhancement of Electrical and Mechanical Properties of SU-8 Photocrosslinked Coatings Containing Polypyrrole-graphene Oxide Nanoparticles
}

\author{
Mehdi Sharif and Behzad Pourabas* \\ Department of Polymer Engineering, Nanostructured Materials Research Center, \\ Sahand University of Technology, Tabriz 51335-1996, Iran \\ Tel: +98413345 9083; Fax: +98413344 4313 \\ *Corresponding author: pourabas@sut.ac.ir
}

\begin{abstract}
In the present study, photo-curing of polypyrrole decorated graphene oxide nanoparticle (PPy-GO) filled SU-8 photoresist and its effect on the electrical and mechanical properties of nanocomposite were investigated at different loading level of PPy-GO. Chemical structure of SU-8 and its nanocomposites before and after UV irradiation have been characterized using infrared spectroscopy. A significant increase of electrical conductivity was observed as a function of filler content in the nanocomposites confirming the conducting nature of the polymeric coating with incorporation of PPy-GOs. The real permittivity was observed to increase with increasing the PPy-GOs nanofiller loading, and the enhanced permittivity was interpreted by the interfacial polarization. The mechanical properties analysis demonstrated that the hardness has increased 2 time and SEM observations indicated well dispersion of PPy-GO nanoparticles within the cured SU-8 resin matrix.
\end{abstract}

Keywords: photo-curing, SU-8 photoresist, polypyrrole decorated graphene oxide

\section{Introduction}

In the recent year application of photo-curing technique in the development of micro-and nano-technologies has been proven to be of high interest for industrial and academic points of view [1]. Photolithography has traditionally been used to transfer a pattern in a thin film for the preparation of photoresists, but recently it is also used as structural material in micro- and nanotechnologies [2]. In this technology, SU-8 epoxy based negative tone photoresist is extensively employed because of its good thermo-mechanical properties [3]. Furthermore, the good chemical resistance and biocompatibility [4] make SU-8 the material of choice in microfluidic devices fabrication [5]. Electrical and mechanical properties of the UV-cured SU-8 based formulation must be modified in some of applications. This is particularly important in electronic applications where high electrical conductivity is requested in order to develop miniaturized devices.

Addition of carbon-based nanofillers such as graphene, graphite or its derivate such as graphene oxide (GO) can improved electrical conductivity and mechanical properties of polymer composites, due to their unique nanostructures and superior properties [6-8]. Graphene can be synthesized very easily by the chemical reduction of graphite oxide (GO) [9]. Graphene oxide sheets are heavily oxygenated graphene that can alter the van der Waals interactions significantly; and, by functionalization of $\mathrm{GO}$, interaction between $\mathrm{GO}$ and polymer matrix can be improved [10]. For this reason, GO has attracted considerable attention as a nanofiller for the preparation of polymer nanocomposites [11,12]. Electrical conductivity of GO is very poor due to the presence of highly electrical resistant groups like carboxyl, hydroxyl, or epoxy in GO sheets; thus its usage in electrically active materials is limited. Therefore, incorporation of $\mathrm{GO}$ with conducting materials such as conducting polymer leads to the formation of nanocomposites, which have some advantages compared to both components, such as good
Received June 10, 2016

Accepted July 6, 2016 
mechanical strength because of the carbon matrix and excellent electrical conductivity due to the conducting polymer [13-15]. Among the conducting polymers, polypyrrole (PPy) is popular due to its low cost, good electrical conductivity, environmental stability and facile synthesis. Composites based on PPy and GO have shown synergistic properties such as enhancement in electrical conductivity and electrochemical cyclability. However, in most studies PPy-GO composites have been prepared by in situ polymerization technique [16-18].

These preliminary investigations seem to put PPy-GO as a good filler candidate to improve, at the same time, both electrical and mechanical properties of polymeric composites. For these reasons we investigated the effect of the dispersion of PPy-GOs in enhancing electrical and mechanical properties of UV-cured SU-8 resin. PPy-GOs were homogeneously dispersed into SU-8 resin and nanocomposites polymeric films were obtained via UV-irradiation. The photopolymerization process was monitored via FT-IR analysis and the crosslinked films were fully characterized in term of electrical and mechanical properties.

\section{Experimental}

\subsection{Materials}

The SU-8 epoxy based photoresist was used as photocurable resin. The commercially available resin already contains the cationic photoinitiator. PPy-GO was synthesized as previously reported in the literature [11].

\subsection{Sample preparation}

PPy-GO nanoparticles were directly dispersed in the range between 0.5 and $1.5 \mathrm{wt} \%$ in the solution of the epoxy resin by means of Ultra Turrax, a mixing procedure at $30,000 \mathrm{rpm}$ for $10 \mathrm{~min}$ and 10 min in an ultra-sonic bath was carried out to reach a good dispersion of filler in the resin. The formulations were coated on PP substrate. A soft-bake at $80^{\circ} \mathrm{C}$ was performed for $10 \mathrm{~min}$ in order to promote the solvent evaporation. Photo-curing of the formulations was obtained using Fusion lamp ( $\mathrm{H}$ bulb, light intensity on the surface of the sample $150 \mathrm{~mW} / \mathrm{cm}^{2}$, measured with EIT instrument), and a tack-free and free-standing films (thickness $50 \mu \mathrm{m}$ ) were obtained after three passages under the light (velocity of the belt 6 $\mathrm{m} / \mathrm{min}$ ) and they were fully characterized after a post-exposure bake at $80^{\circ} \mathrm{C}$ for $1 \mathrm{~h}$.

\subsection{Sample characterization}

The extent of the photo-polymerization reaction was deter-mined by FTIR spectroscopy (using the instrument Thermo-Nicolet5700). $25 \mu \mathrm{m}$-thick formulations were coated onto silicon wafers and FT-IR spectra were collected before and after UV-irradiation under Fusion lamp. Epoxy groups conversion was followed by monitoring the decrease in the absorbance of the epoxy groups centered at $915 \mathrm{~cm}^{-1}$.

The gel content was determined on the cured films by measuring the weight loss after $24 \mathrm{~h}$ extraction with chloroform at room temperature, according to the standard test method ASTM D2765-84.

The dielectric permittivity was measured by a LCR meter (Agilent, E 4980A) equipped with a dielectric test fixture (Agilent, 16451B) in the frequency range of 40 to $200 \mathrm{KHz}$. The samples used for the tests were disc pellets with a diameter of $40 \mu \mathrm{m}$ and the average thickness was about 2 $\mathrm{mm}$. A piece of rectangular standard Teflon sample with a permittivity of 2.1-2.4 was used for calibration before each test.

The microindentation measurements were carried out at room temperature using a Leitz Microhardness Tester equipped with a diamond square pyramid having an included angle at the tip of $136^{\circ}$ on the surface of plain coated samples. A load of $300 \mathrm{~g}$ was applied for $13 \mathrm{sec}$ from the time of contact of the diamond until the load was removed. The lengths of the diagonals (d) of the indentation formed by the diamond were measured immediately after the removal of the load using a micrometer eyepiece mounted on the microscope. Microhardness was calculated using the equation:

$$
\mathrm{H}=2 \sin 68^{\circ}\left(\mathrm{d} / \mathrm{P}^{2}\right)
$$

where $P$ is the load applied in newtons and $\mathrm{d}$ is the diagonal of the indentation in meters. The average microhardness values presented were calculated from a minimum of 5 indentations evenly distributed over an area of $2 \mathrm{~cm}^{2}$.

\section{Results and discussion}

The aim of this study was the preparation and characterization of SU8/PPy-GO composite UV-cured coatings with enhanced electrical and mechanical properties.

\subsection{UV-curing process}

SU-8 photoresist is a mixture of epoxy novolac resin, a solvent (either $\gamma$-butyrolactone or 
cyclopentane) and a photoacid generator (a triarylsulfonium hexafluroantimonate salt). Photo curing of the SU-8 photoresist occurred in two sequential steps: (1) UV exposure for formation of the photoactivator and (2) post-exposure bake (PEB) to allow the polymerization of SU-8 monomers $[19,20]$. The chemical structures and reactions involved in this process are presented in Fig. 1.

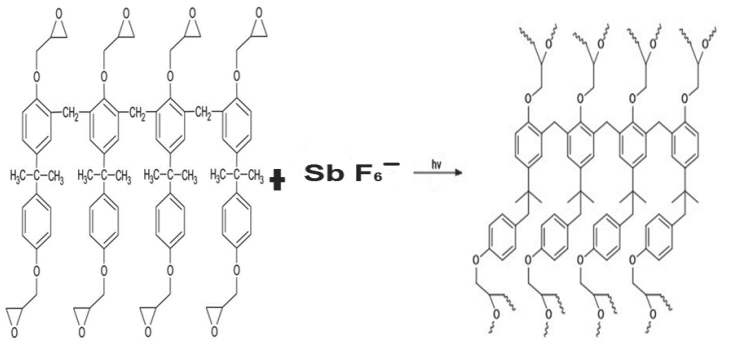

Fig. 1. Chemical structures and photopolymerization reaction $\mathrm{SU}-8$.

The FTIR spectrum of a film of the SU-8 resin or monomer is characterized by an absorption peak at $915 \mathrm{~cm}^{-1}$ assigned to epoxy ring vibration mode and a peak at $1608 \mathrm{~cm}^{-1}$ characteristic of the aromatic ring $\mathrm{C}-\mathrm{C}$ stretch mode (Fig. 2) [21]. The FTIR spectrum of SU-8 photoresist after UV exposure and a PEB $\left(80^{\circ} \mathrm{C} 1 \mathrm{~h}\right)$ demonstrates a peak at $1608 \mathrm{~cm}-1$ identical to that of the resin and photoresist without UV exposure. As expected, the aromatic ring remains after the cross-linking reaction. In contrast, the peak at $915 \mathrm{~cm}^{-1}$ is disappeared suggesting that the epoxy rings are consumed in the cross-linking reaction. A detailed study of the epoxy resins spectra allowed us to check that the photocuring process has been completed in SU-8/PPy-GO nanocomposites. Thus, no absorption band was detected at $915 \mathrm{~cm}^{-1}$, which is consistent with the absence of epoxy groups. Therefore, it can be concluded that fully cured films were obtained after two passages under UV-lamp with an almost complete epoxy group conversion (see data reported in Table 1). Moreover, all the cured samples showed a high gel content value (above 99\%, see Table 1), indicating the absence of any extractable oligomer.

Table 1. Properties of photo-cured SU-8/PPy-GO nanocomposites films.

\begin{tabular}{|c|c|}
\hline Sample & Gel content (\%) \\
\hline SU-8 & 100 \\
\hline SU-8+0.5 wt \% PPy-GO & 100 \\
\hline SU-8+1 wt \% PPy-GO & 100 \\
\hline SU-8+1.5 wt \% PPy-GO & 98 \\
\hline
\end{tabular}

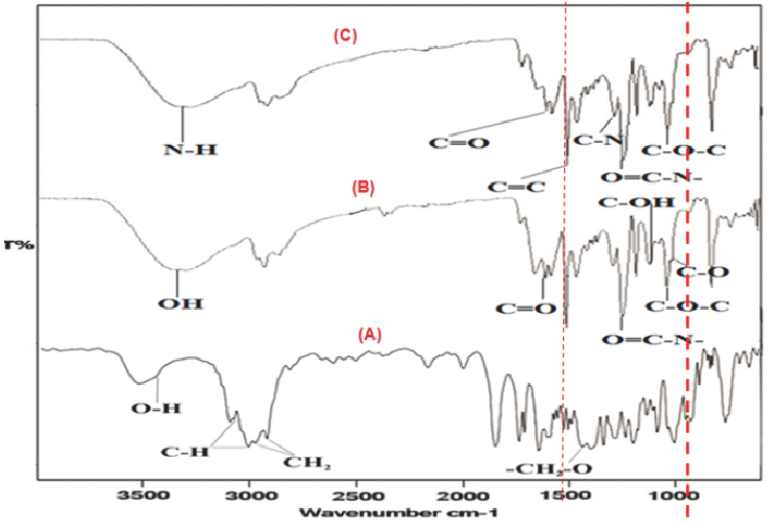

Fig. 2. FTIR spectra of SU-8 resin and photoresist nanocomposite A) SU-8 befor irradiation, B) SU-8 after photocuring, C) photocured SU-8/PPy-GO.

\subsection{Morphological analysis}

Morphological investigations of the UV-cured films were performed by SEM analysis. In Fig. 3 the SEM images of pure SU-8 and SU8/PPy-GO composite containing $0.5,1$ and $1.5 \mathrm{wt} \%$ of PPy-GO are reported. From the pictures it is possible to observe that PPy-GO nanoparticles were uniformly dispersed throughout the polymer matrix. It can be observed that particle size of PPy-GO phase increased with the increase of PPy-GO concentration and the filler particles appear to be completely embedded in the SU-8 matrix. This morphological distribution of fillers should be convenient for electrical conductivity and enhanced mechanical properties of the composites.

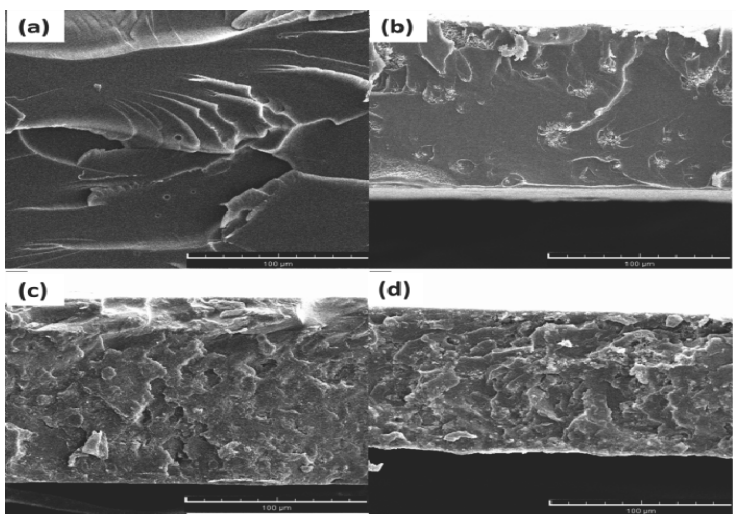

Fig. 3. SEM micrograph of (a)SU-8 B1 (b)SU8/PPy-GO $0.5 \%$ (c) SU8/PPy-GO $1 \%$ and d) SU8/PPy-GO $1.5 \%$.

\subsection{Electrical properties}

The dielectric property of pure SU-8 and its nanocomposites were studied with the variation of real permittivity $(\varepsilon ')$, imaginary permittivity $(\varepsilon ")$ and dielectric $\operatorname{loss}(\tan \delta)$ as a function of the 
frequency. As shown in Fig. 4, for all the samples, both $\varepsilon$ ' and $\varepsilon$ " decrease with increasing frequency, which is typical dielectric relaxation and can be attributed to the reorientation of the permanent molecular dipoles [22].
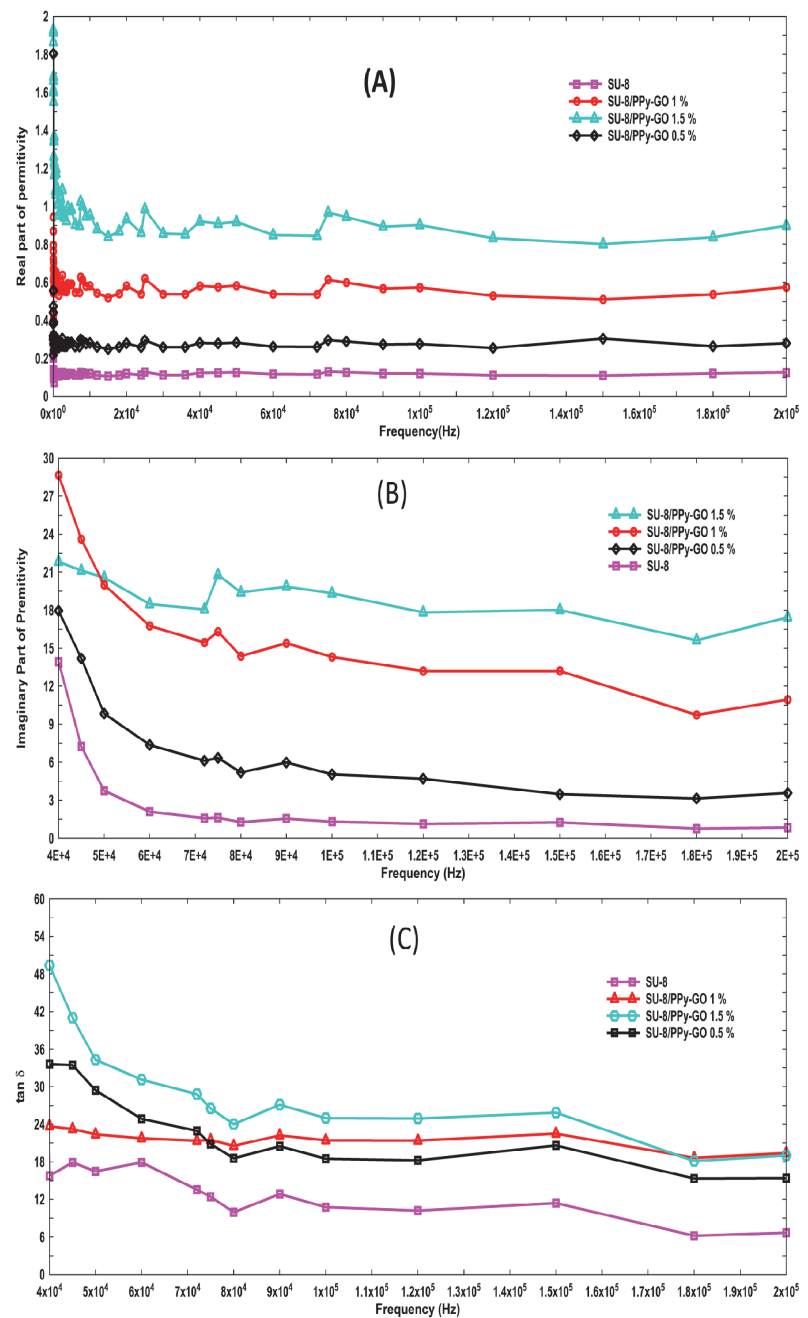

Fig. 4. (A) Real permittivity ( $\left.\varepsilon^{\prime}\right)$, (B) imaginary permittivity $(\varepsilon ")$, and $(\mathrm{C})$ dielectric loss $(\tan \delta)$ of cured pure SU-8 and its nanocompoaite with different loading levels of PPy-GO.

The orientation of dipolar groups under an alternative electric field depends on the frequency value. At lower frequency, the dipolar functional groups can orient themselves and a high permittivity value is observed; however, at increased frequency, the dipolar groups cannot orient at the same rate of the alternating electric field and thus cause a reduced permittivity [23]. All the SU-8/PPy-GOs nanocomposites show higher $\varepsilon^{\prime}$ and $\varepsilon^{\prime \prime}$ than pure SU-8 and the values of both permittivities increase with increasing the loading of the PPy-GO nanofillers. The enhanced permittivity was widely observed in the nanocomposites and was attributed to the interfacial polarization in which the charge carriers were blocked at the internal surface or at interfaces between the matrix and the fillers leading to an increased permittivity [24,25]. In the SU-8/PPy-GOs, although the protons (hydrogen ions) provided by the doping acid can move along the PPy chains, these charge movements will be hindered by SU- 8 resin, thus, a large number of space charges will accumulate at the interface of PPy and SU-8 and result in a larger interfacial polarization. The observed higher $\tan \delta$ value of the SU-8/PPy-GOs than that of pure SU-8, Fig. $4(\mathrm{C})$, is associated with the free charge motion difference, indicating an interfacial polarization occurring in the SU-8/PPy-GOs [26].

\subsection{Microhardness}

As microhardness measurements can be performed quite rapidly on a small sample without destroying it, such measurements certainly may have a potential advantage as compared to investigations using full-scale tensile or impact tests. The microhardness values obtained for the SU-8 and SU-8/PPy-GOs nanocomposites are tabulated in table 2 . The results show that addition of the PPy-GO greatly increased the microhardness $(160.5 \mathrm{MPa})$ with regard to the neat SU-8 $(=81.6$ $\mathrm{MPa}$ ). This hardness increase can be related to the presence of reinforcing filler and hindering effect of polymer chain mobility induced by the PPy-GO layers dispersed within the polymeric matrix. This difference reflects the tremendous improvement in mechanical properties. It could be assumed that the morphology of nanocomposites and, in particular, the good PPy-GO dispersion and higher level of exfoliation are the reasons for the high values of the microhardness. It can be seen that at PPy-GO concentration $(>1 \mathrm{wt} \%$ ) hardness decreased which related to the PPy-GO dispersion state in SU-8 matrix. This is similar to another study which reported that poor filler dispersion and even filler agglomeration lead to lower microhardness values of polymer/carbon nanotube composites [27].

Table 2. Microhardness (H) of SU8 and its nanocomposite.

\begin{tabular}{|c|c|}
\hline Sample & H (MPa) \\
\hline SU-8 & $81.6 \pm 26$ \\
\hline SU-8+0.5 wt \% PPy-GO & $103.5 \pm 35$ \\
\hline SU-8+1 wt \% PPy-GO & $160.3 \pm 23$ \\
\hline SU-8+1.5 wt \% PPy-GO & $128.5 \pm 43$ \\
\hline
\end{tabular}




\section{Conclusions}

PPy-GO was dispersed into photocurable SU-8 in order to enhance both electrical and mechanical properties of UV-cured composite. Fully crosslinked hybrid network was obtained by using a very high light intensity lamp. The UV-cured materials were fully characterized. Morphological investigation showed that PPy-GOs were uniformly dispersed throughout the polymer matrix. The electrical conductivity of the polymer was observed to increase as a function of filler content in the nanocomposites, confirming the electrical conducting nature of the polymer with incorporation of PPy-GO. In conclusion, the high efficiency of PPy-GO as conductive filler in improving electrical conductivity and mechanical properties through the polymer composite has been demonstrated.

\section{Acknowledgement}

The authors acknowledge the main financial resource received from Iran National Science Foundation; contract No. 91004398. Moreover, part of the expenses was supplied by Iran Nanotechnology Initiative Council.

\section{References}

1. W. H. Teh, U. Durig, U. Drechsler, C. G. Smith, and H. J. Guntherodt, Appl. Phys., 97 (2005) 054907.

2. P. Abgrali, V. Conedera, H. Camon, A. M. Gue and N. T. Nguyen, Electrophoresis, 28 (2007) 4539.

3. A. Del Campo and C. Greimer, Micromech. Microeng., 17 (2007) 81.

4. B. A. Weisenberg and D.L. Mooradian, Biomed. Mater. Res., 60 (2002) 283.

5. P. Abgrall and A. M. Gue, Micromech. Mircoeng., 17 (2007) R15.

6. D. Li and R. B. Kaner, Science, 320 (2008) 1170.

7. S. Stankovich, D. A. Dikin, G. H. B. Dommett, K. M. Kohlhaas, E. J. Zimney, and E. A. Stach, Nature, 442 (2006) 282.

8. W. Lu, H. Lin, D. Wu, and G. Chen, Polymer, 47 (2006) 4440.
9. N. Liu, F. Luo, H. Wu, Y. Liu, C. Zhang, and J. Chen, Adv. Funct. Mater., 10 (2008) 1518.

10. S. Rattana, N. Chaiyakun, N. Witit-anun, P. Nuntawong, S. Chindaudom, C. Oaew, and P. Kedkeaw, Proced. Eng., 32 (2012) 759.

11. M. Zare, M. Sharif, and A. Kashkooli, Polym. Plast. Technol. Eng., 53 (2014) 1392.

12. M. Amani, M. Sharif, A. Kashkooli, N. Rahnama, and A. Fazli, RSC Adv., 5 (2015) 77723

13. Z. Ming, Y. Zhai, and S. Dong, Anal. Chem., 14 (2009) 5603.

14. W. Yang, K. Ratinac, S. Ringer, P. Thordarson, J. Gooding, and F. Braetet, Chem. Eng., 49 (2010) 2114.

15. D. W. Wang, F. Li, J. Zhao, W. Ren, Z. Chen, and J. Tan, ACS Nano, 22 (2009) 1745.

16. S. Bose, T. Kuila, M. Uddin, N. H. Kim, and A. Lau, Polymer, 51 (2010) 5921.

17. P. Wang, Y. Zheng, and B. Li, Synth. Met., 166 (2013) 33.

18. C. Bora and S. K. Dolui, Polymer, 53 (2012) 923.

19. R. S. Lima, C. Leão, P. A. Gomes, A. M. Monteiro, M. H. O. Piazzetta, A. L. Gobbi, L. H. Mazo, and E. Carrilho, Electrophoresis, 34 (2013) 2996.

20. F. Niklaus, G. Stemme, J. Lu, and R. Gutmann, Appl. Phys., 99 (2006) 28

21. T. L. Tan, D. Wong, P. Lee and R. S. Rawat, Appl. Spectrosc., 58 (2004) 1288

22. M. Rahman, C. W. Hsieh, C. T. Wang, B. R. Jian, and W. Lee, Dyes. Pigm., 84 (2010) 128.

23. S. Singha and M. J. Thomas, IEEE Trans. Dielectr. Electr. Insul., 15 (2008) 12.

24. C. D. Liu, S. N. Lee, C. H. Ho, J. L. Han, and K. H. Hsieh, Phys. Chem. C, 112 (2008) 15956.

25. A. Schönhals, H. Goering, F. R. Costa, U. Wagenknecht, and G. Heinrich, Macromolecules, 42 (2009) 4165.

26. J. Lu, K. S. Moon, B. K. Kim, and C. P. Wong, Polymer, 48 (2007) 1510.

27. C. Hui, F. Yan, and Q. Xue, Mater. Sci. Eng. A, 364 (2004) 94. 